\title{
Types of Inferences in the Comprehension of Metaphor
}

\author{
Ramona Pistol \\ Middlesex University, London, UK
}

\begin{abstract}
This paper focuses on the cognitive processes involved in the comprehension of metaphors. It builds on current studies in cognitive linguistics by proposing an amendment to the theoretical idea that there are two routes for comprehending metaphors. It presents an account which is underlined by concept adjustment in all types of metaphorical instances, with the only difference being in the degree of the inferences that it requires. In this paper I claim that a conscious level of processing is involved in the comprehension of some metaphors in order to determine the speaker's meaning. I ground my discussion squarely in the distinction between types of inferences developed by Mercier and Sperber (2017).
\end{abstract}

Index Terms - metaphors, conceptual metaphor theory, relevance theory, cognition, contextual assumptions, inferences, reasons, argumentative theory of reasoning

\section{INTRODUCTION}

Although numerous studies have focused on metaphorical language and cognition, the cognitive processes involved in metaphorical comprehension are still open to debate. Metaphors have been found to abound in everyday communication. Contemporary cognitive approaches to language have turned the view of metaphor from being a mere rhetorical and poetic form of language (e.g. Aristotle) to being spontaneously used in everyday communication (cf. e.g. Gibbs 1994). The abundance of metaphorical instances varies from conventional and familiar forms, such as "bright student", "He is a snake/shark/lion/pig/butterfly", "You are wasting my time", "She is a Bridget Jones", to those highly poetic and extended over a length of text such as "There were sheep in the distance wrapped in mist, the trees wearing mist as scarves. The light curdled like old milk" (Lucy Wood, Weathering), "As this life is not a gate, but the horse plunging through it". (Jane Hirshfield, The Tongue Says Loneliness). The highly conventional and semi-lexicalised metaphorical forms often go unnoticed by language users. In contrast, the creative metaphorical language accentuates the awareness of two concepts being used and this is often referred to in the literature as "metaphoricity". Camp (2008) calls this recognition stage "the intuitive gap" because communicators are aware of the tension between the literal and the figurative meaning and, as a result, they invest more conscious effort in the interpretation process.

This perceived distinction, together with the belief that the creative types of metaphors give rise to more vivid imagery and rich poetic effects by exploring in more depth the perceptual and affective information and the findings that some components prime each other, has lead Carston $(20009,2010,2012)$ to put forward the idea that there are two cognitive processes that metaphorical comprehension requires, and these are broadly given by the two types mentioned above. The first is the standard relevance theoretic account that involves a lexical adjustment process (for details see Wilson and Carston 2007). The latter process is one where the literal meaning is metarepresented as a whole and is subject to slower and more reflective pragmatic inferences. When she describes the dual processing model, Carston (2010) makes a very short reference to Sperber's reflective inferences in relation to the metarepresentational level. Sperber (1999) suggests two types of inferences in human cognition which can be applied to language comprehension in general and perhaps to the two distinct ways suggested by Carston. This distinction at an inferential level is developed by Mercier and Sperber $(2017,2011)$ who argue that reasoning involves the process of metarepresenting reasons, but most importantly, they claim that reasons play a significant role in the after the fact explanation and justification of our intuitions, and not in the process of intuitive inference itself.

The present paper investigates this theoretical assumption in relation to metaphorical processing. It aims to address the question whether there are more than one ways of understanding metaphors. Subsequently, are all types of metaphors subject to the same inferential processes? To answer these questions, I review two theoretical frameworks of the underlying mechanisms in metaphorical comprehension, followed by a breakdown of the reasoning mechanisms involved. I look at under what circumstances we can distinguish between types of metaphors and whether the comprehension processes are specific to only metaphorical language. I will then go to explore the extent to which these psychological mechanisms can draw a distinction between types of metaphors.

\section{COGNITIVE ACCOUNTS OF METAPHOR COMPREHENSION}


In addition to the open debate on whether metaphor is a matter of thought as argued by cognitive linguists or arises in language use as claimed by relevance theorists, several theorists favour distinctive mechanisms that underlie metaphor comprehension in regards to their familiarity and novelty (e.g. Bowdle and Gentner 2005, Giora 2003, Glucksberg 2008, Steen 2008). According to Bowdle and Gentner's (2005) career of metaphor hypothesis, comprehension of familiar metaphors such as Faith is an anchor is directly recovered from memory via categorisation, while novel metaphors such as $A$ fisherman is a spider require comparison-based processes to generate a mental image as the result of mapping the relational structures of spider into that of fisherman. Glucksberg's (2008) categorisation based account also allows for comparison, but under different circumstances. Glucksberg and Haught (2006) point to the fact that in a comparison form, the metaphor vehicle refers to the literal concept, whereas in a categorical form the reference is an abstract (metaphorical) category. Hence, there is a strong idea that comprehension process of familiar metaphors is different from the one involved in novel uses. This is at odds with the relevance theoretic unified account that places metaphor comprehension at the end of a continuum with other cases of loose use of language such as approximation, category extension and hyperbole.

It is commonly agreed now that literal meaning does not have unconditional priority and metaphorical meanings are automatic and mandatory (Gibbs 1994, Glucksberg 2008). This idea is particularly strengthened by truth-conditional account of relevance theory (Carston 2002, Sperber and Wilson 2008). This view presents metaphor comprehension as including the creation of an occasion specific concept that comprises of the relevant meaning. On this account, the literal meaning only acts as a point for pragmatic processes, allowing processing of relevant meaning only. However, Wearing (2014) suggests an analogical reasoning process for comprehension of category crossing metaphors and novel metaphors to address the inadequacy of the ad hoc concept to trigger an appropriate figurative interpretation.

Carston $(2009,2010)$ draws a loose distinction (the distinction is not clear cut) between "ordinary" metaphors which are familiar metaphorical uses such as (1) and (2) and "literary" metaphors which are creative and carefully crafted such as (3) and (4) and suggests two different comprehension mechanisms to match this distinction.

1. The assignment was a breeze.

2. A belief that the sword or the gun or the bomb or the propaganda machine is the ultimate arbiter of what's true and what's right. (Obamas's farewell speech 2017)

3. The tongue says loneliness, anger, grief, but does not feel them. (Jane Hirshfield - "The tongue says loneliness")

4. There was a distance, but the distance was benign, brought about simply by a removal that was physical and not the result of estrangement or over-familiarity. (Anita Brookner, At the Hairdresser's)

The first is the standard relevance theoretic account of lexical meaning adjustment which is quick and local and results in the creation of an ad hoc concept. In addition, complex and truly evocative metaphors such as (3) and (4) call for a slower, more reflective process in which the literal meaning of the metaphorically used expression plays a more central role.

Before assessing the implications of Carston's dual processing model, I will first briefly consider some recent suggestions on the notion of concepts which challenges the traditional view that words encode concepts. Recent theoretical discussions over concepts by Allott and Textor (2011) and Carston (2016) tend to favour a more schematic representation. Relevance theory holds a computational view where words encode atomic concepts (addresses in memory). However, Carston's $(2012,2013)$ envisages a non-conceptual view within RT where conceptual expressions "point" to something looser than a stable conceptual space, a "template" for the construction of a fully propositional conceptual structure. The idea is that words activate bundles of senses/concepts that are associated with them and others that are not yet fully established as stable senses. However, an evaluation of the idea that lexical meaning of words is not a full-fledged concept is beyond the scope of this work. As Carton (2016) points out, even if the idea that words do not encode concepts proves right, it doesn't necessarily imply that it will fundamentally affect the lexical adjustment/modulation since, on the relevance theory account, decoding of a lexical concept is done through activating its encyclopaedic entries which capture general and contingent knowledge, including images about the denotation of the concept. Similarly, Allott and Textor (2011) argue for a view of ad-hoc concepts as clusters of information relevant for inferences. Their point is that this is reflected in their activation and the fact that they are constructed without much conscious reflection.

Carston's additional interpretation route does not involve forming ad hoc concepts. Instead, it entails several other stages. Interpreters entertain the vivid imagery and the literal meaning as a whole and frame or metarepresent it as descriptive of an imaginary world. Carston exemplifies this point with the following example:

Depression, in Karla's experience, was a dull, inert thing - a toad that squatted wetly on your head until it finally gathered the energy to slither off. The unhappiness she had been living with for the last ten days was a quite different creature. It was frantic and aggressive. It had fists and fangs and hobnailed boots. (Zoë Heller, The Believers, 2008, p. 263)

Carston believes that creating a succession of ad hoc concepts such as TOAD*, CREATURE*, FISTS*, FANGS* for each metaphorical instance would create a lot of effort for insufficient cognitive reward because of the high activation of the closely associated literal meanings, i.e. priming. The vivid accompanying imagery is used for inferential processes and will attract interpreters' attention. Th literal meaning is held in mind (the literal meaning remains activated well beyond the point the metaphorical interpretation has been recovered in any types of metaphors) and used 
to create an imagined world that will be then used to derive implications to arrive at the intended meaning. To exemplify with the above passage, the set of conceptual representations of an imagined world of repulsive amphibious creatures with different kinds of characteristics (some sitting inertly on human heads, some kicking and biting) are framed or metarepresented and subjected to reflective inferential processes. The framing level is the stage where the literal meaning (or say: sets of literal representations) is held for a further process of inspection of its conceptual properties (implications, associations). Thus, the metarepresentational level acts as a filter for a reflective inferential processing which, in turn, inspects and judges which implications apply to the actual world, that is to be true. Hence, from the patently false representations of depression as a toad and grief as a creature, we derive implications that accord with our experiences of relevance.

The factors that Carston believe can trigger the "imaginary world" interpretation route include the novelty, complexity and creativity of the metaphorical expression, an intensified role of the literal meaning, the supposition that some components prime each other, the communicative context and also individual's preferences towards a reflective practice. Also, she argues that in extended metaphors, the literal meaning becomes highly activated and accessible because the words have been semantically primed and reinforced and thus, they become much more accessible than the pragmatic ad hoc process, which is believed to be more effortful. What follows from Carton's account is that the supremacy of the literal meaning over a fast and subconscious process of modulating a concept does not only generate a conscious process that helps interpreters reflect upon a possible meaning, but it can also direct them to manipulate information in order to establish how the meaning relates to them, making it a process of judgement of information. This brings Carston's view closer to how Mercier and Sperber (2009, 2010, 2017) describe reflective inferences. An obvious similarity is the description attributed to reasoning by Mercier and Sperber who claim that reasoning is a special form of inference at a conceptual level. This type of inference underlines not only a consciously produced new mental representation or conclusion but also a consciously entertained representation that warrant it. Consciousness seems to play a very important role in this type of inferences and metarepresentation. Mercier (2017) discusses the similarities of the metarepresentational module to Stanovich's (2013) notion of "decoupling" that is attributed to system 2 processes (see dual process accounts of human reasoning). For Mercier, cognitive mechanisms of mindreading and pragmatics rely on "decoupling".

Carston's claim that the literal meaning is held without being adjusted in any way poses several questions. What actual properties of the encoded concepts are "held" and not used for concept adjustment? Do hearers hold the most salient feature of the literal meaning together with its contextual implicatures, or will they be looking for the core properties of the concepts? And most importantly, is it the metarepresentational level just the first stage followed by other reflective inferential processes as Carston implies? Carston's account seems plausible to address the shortcomings of a consciously process in arriving at a metaphorical interpretation (especially since this has been overlooked in relevance theory), but it doesn't address the possibility of reflecting at the conscious level when creating an ad hoc concept.

Ultimately, reflection is a metarepresentational process itself as described by Sperber (1997) and Mercier and Sperber $(2009,2011)$. They consider reflective inferences to involve the representation of reasons. Reasons are examined in order to reach a reflecting conclusion which then needs to be accepted. A very similar line of thought is indicated by Carston when she explains that the deliberately created imaginary world (literary false conceptual representations and their imagery are metarepresented and subject to a more reflective inferential processes) is subjected to "slower, more reflective interpretive inferences that separate out implications that are plausibly speaker-meant". The reason for this is that interpreters inspect and judge which implications are true. This process gives the slow and effortful characteristics of the additional comprehension route for metaphors. The stage in Carston's account where hearers inspect the implications is a stage of reflection. At first glance, the reflective process proposed by Carston seems to involve the idea put forward by Mercier and Sperber $(2009,2011,2017)$ that the reflective inferences involve a conscious stage of creating a new mental representation, but at the same time consciously entertaining previously held representations that warrant it. It might be also the case that the vivid imagery in literal metaphors is an element that triggers the transition to a consciousness stage. Ellis and Newton (2010) assume that consciousness corresponds to higher order processes and it can allow the interpreter to focus more on some parts that make up the whole. Carston focuses on the important role of the literal meaning in the second route, but there is a possibility that interpreters become conscious of the need for lexical concept adjustment to fit the interpretation. It might be effortful in a series of semantically primed metaphorical vehicles to pragmatically infer the occasion specific concept of each, given the extended nature of the metaphor and the importance of contextual information, but there is also a possible conscious adjustment of the concept during which interpreters are searching for those encyclopaedic entries that can fit the metaphorical interpretation. Metarepresenting the ad hoc concept at this stage would also incorporate the producer's intentions which are part of the overall metaphorical interpretation. Thinking of the literal meaning of a word and being aware that the meaning is metaphorical adds to the cognitive load, which aligns to Carston's view that the reflective process is more demanding in terms of effort. In the next section I consider the phenomenon of metarepresentation and its role in human cognition.

\section{B. Metarepresentation and the Modular Mind}

The ostensive-inferential framework to communication proposed by relevance theory (Wilson and Sperber 2002) highlights the importance of interlocutors to understand each other's communicative intentions in order to arrive at an 
appropriate interpretation. These assumptions about somebody else's intentions are the outcome of inferential processes triggered by decoding linguistic elements and contextual clues. Recognising these intentions and reasoning and making inferences about another's mental states (thoughts) (assessing understanding of false beliefs) is made possible by the Theory of mind (ToM) ability which accommodates our mindreading ability. This ability allows us to represent a representation, hence metarepresent. There is a common agreement among psychologists that much of our use of language is subject to metarepresentational skills as shown by Papafragou (2002). One condition for a metarepresentation to take place is to have a constituent that refers to the property of being a representation (or some other representational property of the representation, like being a belief, having a certain content or truth condition, referring to some particular, etc.). For example, a primary representation of It is raining or The fridge is empty represents a speaker's own thought. It becomes a first order metarepresentation in the following utterance: John says/believes/wants to say that it is raining/the fridge is empty. The addressee attributes the utterance to the speaker and thinks about the speaker's intentions and possible feelings, and therefore metarepresenting somebody's utterance and thought. A third order metarepresentation will look something like John intends me to believe that he believes/wants to say that it is raining/the fridge is empty. In regular mind reading a single level of metarepresentation is generally enough but as Wilson points out, inferential comprehension typically involves several layers of metarepresentation. Wilson (2000) shows that metarepresentation can be infinite, but it stops when the expectation of relevance is achieved. But most importantly, this ability gives us the capacity to distinguish a real from an apparent property of the world

Our mindreading ability is one of the many modules (e.g. decoding and pragmatic module) assumed by the massive modularity hypothesis (Sperber 1994, 2001, 2005, Pinker 1997, but see Carruthers 2006), according to which the mind is a complex system of modules. A module is a dedicated inferential mechanism that contains special-purpose inferential procedures. Sperber and Wilson (2002) believe that within the overall mind-reading module, there has evolved a specialised sub-module dedicated to comprehension, with its own proprietary concepts and mechanisms. These are fast mechanisms which automatically compute a hypothesis about the speaker's meaning. A metarepresentational capacity in adults with no deficit in cognition allows interpreters to deal simultaneously with mismatches and deception.

Metarepresentational ability deficit is connected to lack of metaphor interpretation ability as shown by several studies with autistic and schizophrenic people conducted by Happe (1995) and Baron-Cohen (2003, 2006). The results of Happe's $(1993,1995)$ study show that metaphor requires only a first order theory of mind, and not the ability to attribute thoughts about thoughts to others. Wilson and Sperber (2012) share this claim as their account presents metaphor like instances of ordinary literal speech i.e. as expressing a thought and therefore not involving metarepresentation, in comparison to irony which is seen as requiring the recognition of a thought about an attributed thought (a higher-order metarepresentation). Yet, Wearing (2014) suggests that autistic people follow the ad hoc concept route, but they fail to take into account speaker's beliefs and most importantly, to take the speaker, rather than the world to guarantee the relevance of the utterance. Wearing is right in making this assumption as evaluation of certain type of incoming information is a representational activity. Recent studies by Camp and Carston tend to direct towards something similar to a metarepresentation level in more creative metaphors. Camp (2006) draws attention on the relationship between types of metaphors and their implications for interpretation. In familiar metaphors, such as John is a bulldozer, the role of the speaker's intentions in determining the metaphorical meaning is quite clear. In literary metaphors, aesthetic is important in the relationship between producer and recipient. Aesthetic provides the means to influence meaning on one hand and consider non-semantic features on the other. As Camp remarks, a writer is not aiming for a specific proposition, but he invites the readers to a "free play of ideas" by choosing to use a literary metaphor. For instance, in interpreting example (3) The tongue says loneliness, anger, grief, / but does not feel them. (Jane Hirshfield - "The tongue says loneliness") the writer's interpretative intentions which are still to be discovered are important for a full grasp of the relevant metaphorical meaning. Vivid images and emotions here are a powerful part of the metaphorical meaning. As Camp (2008) puts it, "metaphors employ a characterization associated with one subject as a perspective or frame for thinking about something else, with a felt awareness of the gap between them" (Camp 2008: 18). Carston shares that idea and identifies a level of reflection where the literal meaning is held and the process of creating an imaginary world is taking place. Her point is that some instances of metaphorical use involve an extra layer of metarepresentation of an attributed content or form.

In terms of metarepresentation (3) The tongue says loneliness, anger, grief, / but does not feel them could be formulated as follows:

(a) I know [that the author wants to make me think that [the tongue says loneliness.

Here, interpretation would include altering the interpreter's perception and beliefs and consider the author's intentions. Breham (2012) states that updating one's description of the word in case of conflicting data or expectations includes metarepresentations concerning proper usage. I believe that what is happening in this metaphorical case at the level of thinking is that information is assessed for credibility. The readers know that the tongue cannot say words, but they are also aware that the expression is a metaphor, where one thing is referred to in terms of another. I believe that what is happening here is an evaluation of the plausibility and acceptability of interpretative hypothesis, which is in line with Mercier and Sperber's $(2009,2017)$ distinction between intuitive and reflective inferences which I further detail in 
the next section. The distinction that I will present between two types of inferences focuses on their characteristics and how they vary across comprehension in general.

\section{INTUITIVE AND REFLECTIVE INFERENCES}

Mercier and Sperber $(2008,2017)$ approach human reasoning from an evolutionary psychological perspective which provides the most suitable explanation for why humans reason and set out to examine its cause and process. Their central claim is that reasoning has two main functions: justifying and evaluating arguments. To do so, it uses a special mechanism which has the same core procedures as the rest of the mechanisms used in human thinking. This clearly seems to question the validity of both classic and more recent reasoning accounts. Reasoning is presented as involving higher-order intuitions based on lower-order intuitions which support some conclusions. Contrary to other beliefs, it is limited to only instances of trying to convince others or being convinced by others.

They argue for an evolutionary and modularist view of reasoning. Hence, reasoning is a module which is metarepresented because it draws intuitive inferences about reasons. This argument is made possible by using the tenets of the massive modularity hypothesis which is a model of the human mind (see Sperber 1994, 2001). An important rationalisation for the modularity idea lies in the inferential model of thinking. Mercier and Sperber (2017) regard inferences as involving a variety of mechanisms and argue that reason is only one of these varieties. They argue that reasons play a central role in the after the fact explanation and justification of our intuitions, not in the process of intuitive inference itself. Reasoning has two main social functions: justificatory and argumentative. Hence, we produce reasons to justify ourselves on one hand, and to convince others by constructing arguments on the other. Yet, this claim opposes the Cartesian view that reasoning leads to better beliefs and decisions by overcoming errors (e.g. Kahneman 2003). Inference is carried out through diverse mechanisms and is not a result of logic only. Mercier and Sperber challenge the classical view which presents inferences as a result of logic and argue that they (and cognition more generally) are carried out through diverse mechanisms (or autonomous modules). This is all made possible by our metarepresentational ability. Again, the reason module produces not only intuitive conclusions about reasons, but also reflective conclusions about the things reasons are themselves about.

The main theoretical argument that Mercier and Sperber (2017) sustain is that reasoning is produced by a metarepresentational module - the reason module which has the specific function to produce justifications and arguments. For them, investigating a given module is a matter of relating its procedures to its function because inferential modules aim at providing a special kind of cognitive benefit. Modules in general optimise knowledge and decision making. A metarepresentational module processes representations and attend to properties specific to these representations. The reason module produces justifications and arguments which may have embedded conclusions relevant to all domains of knowledge and action. This makes it a virtual domain general. The specific domain of the reason module is the relationship between reasons and the conclusions they support. Because these reasons and conclusions can be about any topics, the inferences about reasons-conclusions relationships indirectly yield conclusions in all domains, indirectly providing a kind of virtual domain-generality.

Using their evolutionary approach, Mercier and Sperber $(2009,2011,2017)$ argue that reasoning is a type of inference. Inference is a function that is likely to be carried out through a variety of inferential modules. For Mercier and Sperber, inferences can vary from wholly unconscious, automatic and fast (which they refer to as intuitions) to more conscious or likely to become conscious at one point which are deliberate, and very slow (which are referred to as intuitions about reasons). For example, when you are in a cafe and overhear the woman at the table behind saying to the men sitting with her "It's water!" it is difficult to work out the meaning because you lack the relevant contextual knowledge. However, the man in the conversation can infer what the women means without awareness of the inferential process because he has this knowledge. If the man interprets the woman's statement to mean not to worry because the spot on his shirt is just water, then his understanding of this implicit meaning may feel like mere intuitive knowledge. The example illustrates that intuitions are judgements or decisions taken without knowing the reasons that justify them and their production process. On the contrary, in a scenario where the man is thinking about reasons why the woman said to him "It's water!" in a patronising tone of voice, he is likely to be thinking about reasons in favour of the conclusion he reaches. Mercier and Sperber assume that intuitions are delivered by a variety of inferential mechanisms which are more or less specialised. A specialised process implies that each device is attuned to the specific demands of its domain (see Barkow, Cosmides and Tooby 1992). The idea that intuitions are produced through many inferential mechanisms whose processes are unconscious but with conscious conclusions is one of the most important aspects of the authors' main argument.

Mercier and Sperber assert that the role of reasons is to justificatory. They claim that by giving reasons to explain and justify himself, a speaker influences the way other people read his mind, judge his behaviour and indicates that he is likely to evaluate the behaviour of others by similar reasons to those he invokes to justify himself. People create and produce arguments by means of backward inference, from a favoured conclusion to reasons that will support it. Understanding a reason is a metarepresentational task because you need to mentally represent the relationship between at least two representations: the reason itself and the conclusion it supports. One of the most important tools for accomplishing that is language. Language is closely related to our metarepresentational ability and is well adapted to 
represent reasons. For example, a sentence such as "the fact that Molly isn't smiling is a reason to believe that she is upset" metarepresents the relationship between a reason and the conclusion it supports.

\section{INFERENCES IN METAPHORICAL PROCESSING}

When people have relevant pre-existing experiential knowledge, fast recognition processes may provide accurate intuitive responses. These seem to be the same characteristics that are applied to the processes of lexical adjustment of words which underlie the comprehension of ordinary cases of metaphors. Both relevance theorists and Carston (2009, 2010) support the idea that the inferences drawn from creating an ad hoc concept are spontaneous. Comprehension of everyday conversational metaphors such as (1) The assignment was a breeze requires little processing effort and yields limited and predictable effects that underwrites just those inferences that come to mind with the metaphorical interpretation as Wilson and Carston (2007) point out. Narrowing and broadening the sense of BREEZE is done to fit the occasion. The decoded concept BREEZE activates a variety of relevant encyclopaedic properties of different subsets of breezes and enables further conclusions to be drawn. There is a limited variety of senses that can be drawn here. The hearer draws a range of inferences about that particular assignment which include aspects such as being pleasant, gentle, easy. To figure out what (1) means in a conversation the interpreter needs to identify which features associated with breeze might be similar to that particular assignment and also relevant to speaker's intentions. Identifying the relationship, be it of similarity between the properties of the topic and those of the metaphorical vehicle or other correspondence is required for the interpretation of metaphors. Drawing a conclusion from the interaction of assumptions in this case is done through associations and other immediate inputs. It is a straight forward process in the sense that it is a direct output of an inferential module if I take into account Mercier and Sperber's (2017) claim that the modification of belief occurs without the individual's attending to what justifies this modification. Coming out with a surface association between the metaphorical terms and having enough contextual clues to determine the speaker's intentions is enough to meet the expectations of relevance in an everyday face to face conversation. When people have relevant pre-existing experiential knowledge, fast recognition processes may provide accurate intuitive responses. Newly acquired stimuli are assimilated and mapped into pre-existing knowledge structures, being influenced by context and making intuitive processes generating an impression of similarity without intending to do so. For example, a solution to a problem comes to mind quickly because familiar cues are recognised. Conventional metaphors such as (1) usually have their metaphorical meaning listed in dictionaries. Thus, a metaphorical meaning is accessed immediately because the relevant meaning involuntarily comes up. This is possible because the inferential steps involved in narrowing and broadening are subconscious processes.

The alternative processing route proposed by Carston is a reflective comprehension process, thought to be more controlled and reflective than the automatic pragmatic processes engaged in comprehension of familiar metaphors. Again, as with Carston's first route, the additional one shares many of the characteristics of the reflective aspect of thinking described by Sperber (1997) and Mercier and Sperber (2009, 2011, 2017). On their account, reflection is thinking about one's own thoughts, hence a kind of metarepresentational process. Evaluating beliefs is a task that belongs to the argumentation module which is metarepresentational. It indirectly delivers reflective inferences. Discussing the properties of the modules that draw inferences about representations, Mercier and Sperber (2009) say "The conclusion embedded in an output of the argumentation module is disembedded and used as part of the input for another operation of the same module, and this can be reiterated many times" (Mercier and Sperber 2009, p 47).

Considering an example of a literary metaphor, (2) The tongue says loneliness, anger, grief, / but does not feel them, I will try to see what processes are required by drawing the message that is communicated. On the second route, the literal meaning does not just "lingers", but it has a more prominent role given by the fact that it is maintained, developed and represented as material for a reflective pragmatic process that extracts from it relevant implications that are taken to comprise the metaphor's meaning. Unlike the ad hoc route, the metaphorical meaning derived through holding the literal meaning in mind has no explicature and only consists of an array of weakly implicated assumptions that emerge gradually, making it slow and likely analytical. Following Carston's suggestion, says in this context is held for further reflective processes whilst an imaginary world is formed. This means that interpreters create false beliefs which present the tongue as actually saying, that is pronouncing, vocalising, declaring, and so on several words which have negative connotations. The literal meaning in the modulation process is only a point for pragmatic processes and allows processing of relevant meaning only. What it seems to me to happen in example (2) is that hearers become conscious of the literal meaning not in the sense that they hold it for further processes, but because they are evaluating the producer's arguments that challenge their view of the real world. Evaluating arguments triggers slower processes of reflective inferences. But interpreters know that the tongue itself cannot speak and it is just an organ used in articulating speech among other things. This is clearly a stage of hypothetical thinking. What is likely to happen here is that interpreters are searching for a wider range of encyclopaedic properties of the encoded concept during the concept modulation to fit multiple possibilities that will finally lead to the conclusion. Stanovich and Toplak (2012) presume that "the cognitive decoupling" is a central feature for hypothetical thought. Thinking hypothetically involves maintaining a degree of awareness that representations of the real world are not the same as those of imaginary situations. Stanovich (2004) argues that decontextualisation and decoupling skills prevent our representations of the real world from becoming confused with representations of imaginary situations that we create on a temporary basis. This is 
much in line with Carston's assumption that interpreters frame the literal meaning. However, I believe that the conceptual representations that interpreters form are metarepresented in turn to accommodate speaker's intentions and a decoupling in order to generate a serial associative cognition of the type "The author/speaker believes that [The tongue says loneliness, anger, grief, / but does not feel them". The interpreters search for arguments in their reasoning to be able to form new beliefs that can lead to the conclusion that is communicated.

\section{CONCLUSION}

This paper has intended to discuss the theoretical assumption that a perceived difference in metaphorical processing is related to a metarepresentational process of reasons in creating new beliefs. Understanding the speaker's meaning is a process that includes constructing interpretative hypothesis sustained by a series of mental mechanisms and mental modules. This work has supported the argument that novelty of metaphorical instances may trigger a more general reflective mechanism of human cognition which involves the representation of reasons. This, in turn, leads to the suggestion that Carston's two ways of processing metaphors might only be differentiated by an awareness of the reasons needed to reach a conclusion and belong to a much more widespread feature of language which distinguish between intuitive and reflective inferences in communication. The reasons for reaching a decision are argued here to be able to condition cognitive processes involved in metaphorical comprehension.

Undoubtedly, much more work needs to be done to sustain this speculative point of view. A metarepresentational reasoning module is a new paradigm that will need to be studied and tested in other fields and be looked at for implications for general theories of cognition. It remains to be seen whether people are likely to represent reasons in the interpretation of metaphors. In conclusion, I agree that a unified model of inferences as presented by Mercier and Sperber (2017) is a clearer framework which seems well suited for the study of reasoning and metaphorical language.

\section{REFERENCES}

[1] Allot, N. and M. Textor. (2012). "Lexical Pragmatic Adjustment and the Nature of Ad Hoc Concepts", International Review of Pragmatics, 4: 185-208.

[2] Camp, E. (2008). Showing, Telling, and Seeing: Metaphor and 'Poetic' Language. The Baltic International Yearbook of Cognition, Logic, and Communication, 1-24.

[3] Carston, R. (2010a). Lexical pragmatics, ad hoc concepts and metaphor: A Relevance Theory perspective. Italian Journal of Linguistics, 22(1), 153-180.

[4] Carston, R. (2010b). Metaphor: Ad hoc concepts, literal meaning and mental imagery. Proceedings of the Aristotelian Society, $295-321$.

[5] Carston, R. (2012). Metaphor and the literal/nonliteral distinction. In K. a. Allan, The Cambridge Handbook of Pragmatics (pp. 469 - 492). Cambridge: Cambridge University Press.

[6] Carston, R. and Wearing, C. (2011). Metaphor, hyperbole and simile: A pragmatic approach. Language and Cognition, 283312.

[7] Carston, R. (2016) “Contextual adjustment of meaning.” In: The Routledge Handbook of Semantics. Ed. N. Riemer. Abingdon: Routledge.

[8] Evans, J. St. B. T., and Stanovich, K. E. (2013). Dual-Process Theories of Higher Cognition Advancing the Debate. Perspectives on Psychological Science, 8, 223-241.

[9] Glucksberg, S. (2008). How metaphors create categories--quickly. In R. W. Gibbs, Jr. (Ed.), The Cambridge handbook of metaphor and thought (pp. 67-83).

[10] Kahneman, D. (2011). Thinking, fast and slow. New York: Farrar, Straus and Giroux.

[11] Kruglanski, Arie W. (2013). "Only One? The Default Interventionist Perspective as a Unimodel—Commentary on Evans \& Stanovich (2013)." Perspectives on Psychological Science 8 (3): 242-247.

[12] Mercier, H, and Sperber D. (2008). "Intuitive and Reflective Inferences," in In Two Minds: Dual Processes and Beyond, edited by J. Evans and K. Frankish, Oxford: Oxford University Press

[13] Mercier, H and Sperber, D. (2017). The enigma of reason, Harvard University Press, Cambridge.

[14] Romero, E. and Soria, B. (2014). Relevance Theory and Metaphor. Linguagem em (Dis)curso, 489-509.

[15] Sperber, D. and Wilson, D. (2015). "Beyond speaker's meaning." Croatian Journal of Philosophy XV(44): 117-149.

[16] Sperber, D. (2000). Metarepresentations in an evolutionary perspective. In D. Sperber (Ed.), Metarepresentations: A Multidisciplinary Perspective (pp. 117-137). Oxford: Oxford University Press.

[17] Stanovich, K. E. (2011). Rationality and the reflective mind. New York, NY: Oxford University Press.

[18] Wilson, D. (2005). "New Directions for Research on Pragmatics and Modularity," Lingua 115: 1129-1146.

[19] Wilson, D. and Carston, R. (2007). "A Unitary Approach to Lexical Pragmatics: Relevance, Inference and Ad Hoc Concepts," in N. Burton-Roberts (ed.) Advances in Pragmatics, Basingstoke: Palgrave Macmillan, 230-260.

[20] Wilson, D. and D. Sperber. (2012). Meaning and Relevance. Cambridge: Cambridge University Press.

Ramona I Pistol was born in Romania. She has a BA in Philology from the University of Craiova, Romania and a Master in Unity and Diversity in Teaching English as a Foreign Language from the same university.

She taught for 3 years in several schools in Craiova. She is now a PhD student at Middlesex University in London. Her research focuses on cognition and the interpretation of metaphors, reasoning and inferences. 